\title{
Detection of Internal Quality in Seedless Watermelon by Acoustic Impulse Response
}

\author{
B. Diezma-Iglesias , M. Ruiz-Altisent ; P. Barreiro \\ Rural Engineering Department, Physical Properties Laboratory. Polytechnic University of Madrid, E.T.S.I.A. Avda. Complutense s/n. 28040 \\ Madrid. Spain; e-mail of corresponding author: bdiezna(äjiru.etsia.upm.es
}

\begin{abstract}
Recent commercialisation of seedless watermelon varieties relies on the guarantee of a high quality product. Several internal defects may deteriorate greatly this fruit: (a) creases and/or large voids in the flesh, (b) overripeness and (c) bruises due to impact. The objective of this research was to develop a feasible non-destructive procedure for detecting these defects in individual fruits, based on acoustic impulse response.

A device consisting of a microphone, structural elements and a mechanical impact generator was designed and tested. Good and defective seedless watermelons were tested with the acoustic device. Spectral parameters were examined as potential non-destructive predictors of internal disorders. Waveband magnitude parameters, obtained by summing the magnitude of the spectrum between two frequencies in a specified band width (always including between 40 and $500 \mathrm{~Hz}$ ), were the acoustic parameters showing the best ability to detect internal disorders.
\end{abstract}

\section{Introduction}

In Europe, the consumption of watermelon has increased from $87000 \mathrm{t}$ in 1985 to $310000 \mathrm{t}$ in 1997. This increase can be attributed to the introduction of new cultivars of seedless and more tasty watermelon. Nevertheless, these triploid watermelons may have an internal problem called 'hollow heart'. This disorder is a problem most likely to occur early in the season and when growing conditions alternate between wet and dry soil, and hot and cold temperatures. Good pollination is important in preventing this disorder. Too much nitrogen and/or excessive water, especially during fruit set, can encourage hollow heart and bland flavour (Maynard \& Hopkins, 1999). Percentage of affected fruits varies significantly and can range from nil up to more than $50 \%$ of the fruits depending on the batches. Volume of creases and voids varies from $5 \mathrm{ml}$ to more than $1000 \mathrm{ml}$ (Fig. I). While modest hollow volumes may not dramatically affect eating quality or shelf-life, larger volumes reduce freshness and flavour of the watermelons. This is particularly true for watermelons destined for distant markets. In addition, the thickness of the skin makes it difficult to find and segregate fruit affected by bruises. The ability to non-destructively detect hollow heart, overripeness and bruises in individual fruits is highly desirable to minimise the number of affected fruits that will reach the final consumer.

The main purpose of this project was to develop a feasible and non-destructive procedure for detecting hollow heart in individual watermelons. At present, the only method available is the inspection by human experts: the defective watermelon responds with a different resonant sound when tapped. However, this method is subjective and the experts need to be trained for each batch retrospectively. Furthermore, it is not feasible to test each fruit, so that some fruits with internal voids are always found in the market.

Research studies for the non-destructive detection of cracks, notches, or other geometrical changes in structures using changes in the natural frequencies, have been performed by many researchers in different areas (Stubbs \& Osegueda, 1990; Silva \& Gomes, 1994; Kim \& Stubbs, 2003). Crack location models and crack size models have been formulated by relating changes in modal energy to changes in natural frequencies due to damage. 

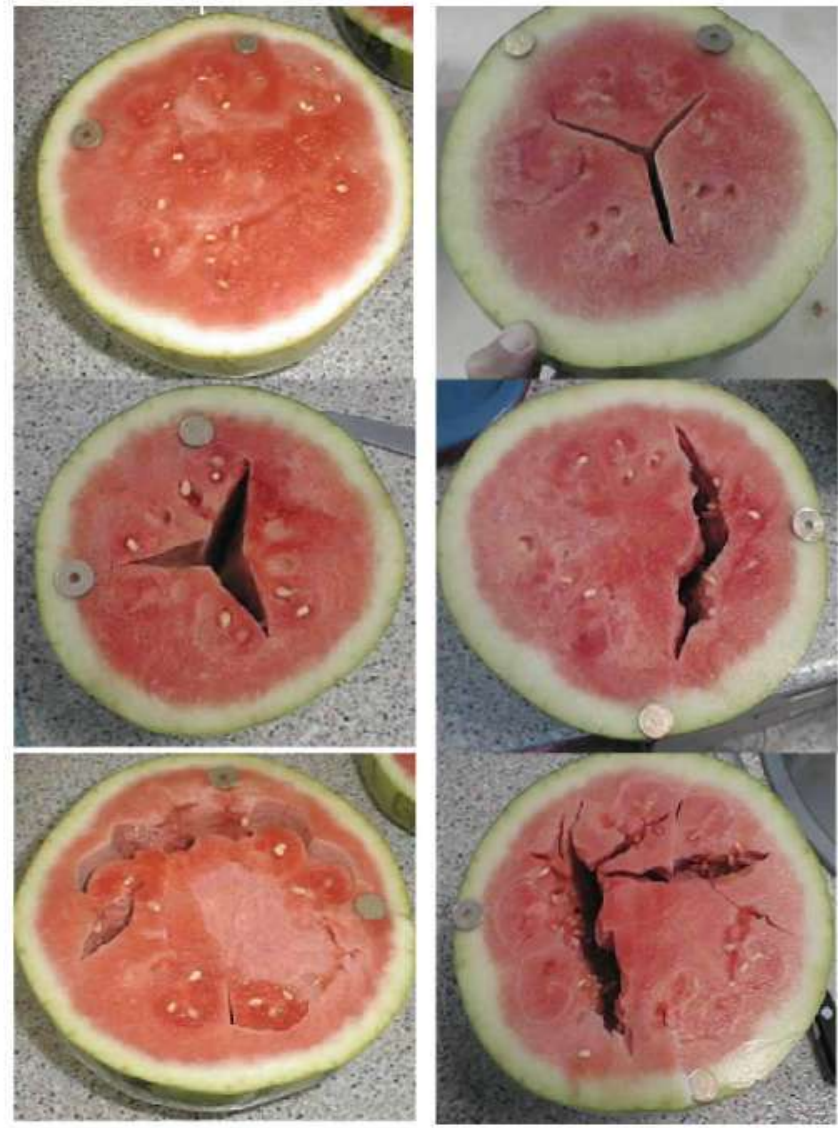

Fig. 1. Watermelons with different levels and types of cracks and hollows

In biological tissues, vibrational behaviour of fruits has been used as an indicator of maturity and postharvest ripeness based on the elastic properties of the tissue. Non-destructive techniques using sonic characteristics of the fruit tissue have been applied for measuring firmness and for detecting internal disorders in several products, such as apples, pears, avocados and melons. Frequently, instruments deliver an impulse to the fruit to produce acoustic vibration (Farabee \& Stone, 1991; Armstrong et al., 1997; De Belie et al., 2000). Different systems are used to sense the vibration of a fruit. Some instruments have piezo-electric sensors, while others employ microphones (De Baerdemaeker et al., 1982; Armstrong et al., 1990; Stone et al., 1996; De Belie et al., 2000).

For the watermelon problem, an initial hypothesis was formulated: the acoustic impact response of a cracked or hollow watermelon is different from the acoustic impact response of a watermelon with good internal quality. The device designed and developed for non-destructively detecting internal creases and/or voids in individual fruits is based on acoustic transmission.

\section{Materials and methods}

\subsection{Instrumental measurements}

Acoustic measurements were taken with a device designed and tested by consecutive phases of approximation to the final solution. The laboratory recording system used to acquire the acoustic impulse information is comprised a prepolarised free-field $12 \mathrm{~mm}$ microphone type $4189 \mathrm{~B} \& \mathrm{~K}$, of a frequency range from $6 \cdot 3$ to $20 \mathrm{kHz}$ and a sensitivity of $50 \mathrm{mV} \mathrm{Pa}^{-1}$. A signal conditioning amplifier NEXUS B\&K supplied power and provided electrical loading to the transducer, amplified the signal, provided appropriate output drive signal and facilitated the selection of the optimum band-pass filters. A microphone preamplifier type 2673 B\&K completed the recording system. The preamplifier amplified the signal from the microphone.

The external system was connected to a computer using an internal Industry Standard Architecture (ISA) personal computer board. It supported the 12-bit analogue/digital converter at a tuneable sample rate up to $40 \mathrm{kHz}$, allowing the user to choose the suitable frequency to obtain the best response, avoiding aliasing for a specific application. It uses eight multiplexed analogy inputs and 24 digital in/out connections.

A user friendly Windows-based software, 'SanSon 1.2 ', was developed for the control of the process and the register of data, providing an easy output to be used with Microsoft Excel. The software shows in the screen the acoustic signal 'time versus intensity' for each test, and saves it in an ASCII file.

A fast Fourier transform (FFT) of the signal was performed to determine the frequency spectrum, and subsequently, the natural frequencies of the watermelons. Sampling at $40 \mathrm{kHz}$ for 4096 points results in a frequency resolution for the FFT of $9.766 \mathrm{~Hz}$. A normalised spectrum was obtained by dividing the magnitude at each frequency by the maximum magnitude of the spectrum (Fig. 2). Different acoustic parameters were evaluated for spectral characterisation: resonant frequency, maximum amplitude of the spectrum and band magnitude (BM) of the acoustic spectrum. The frequency resolution for the spectrum $(9.766 \mathrm{~Hz})$ is not very narrow. Therefore it is difficult to obtain a good segregation based on the resonant frequency, unless higher differences than the resolution occur between hollow and sound watermelons. This handicap may be overcome by defining the integral in the spectrum or BMs. In order to address the optimal $\mathrm{BM}$, several intervals were defined with slight differences between them. The value of the BM obtained by summing up the normalised spectrum magnitude between the encompassing frequencies and dividing by 

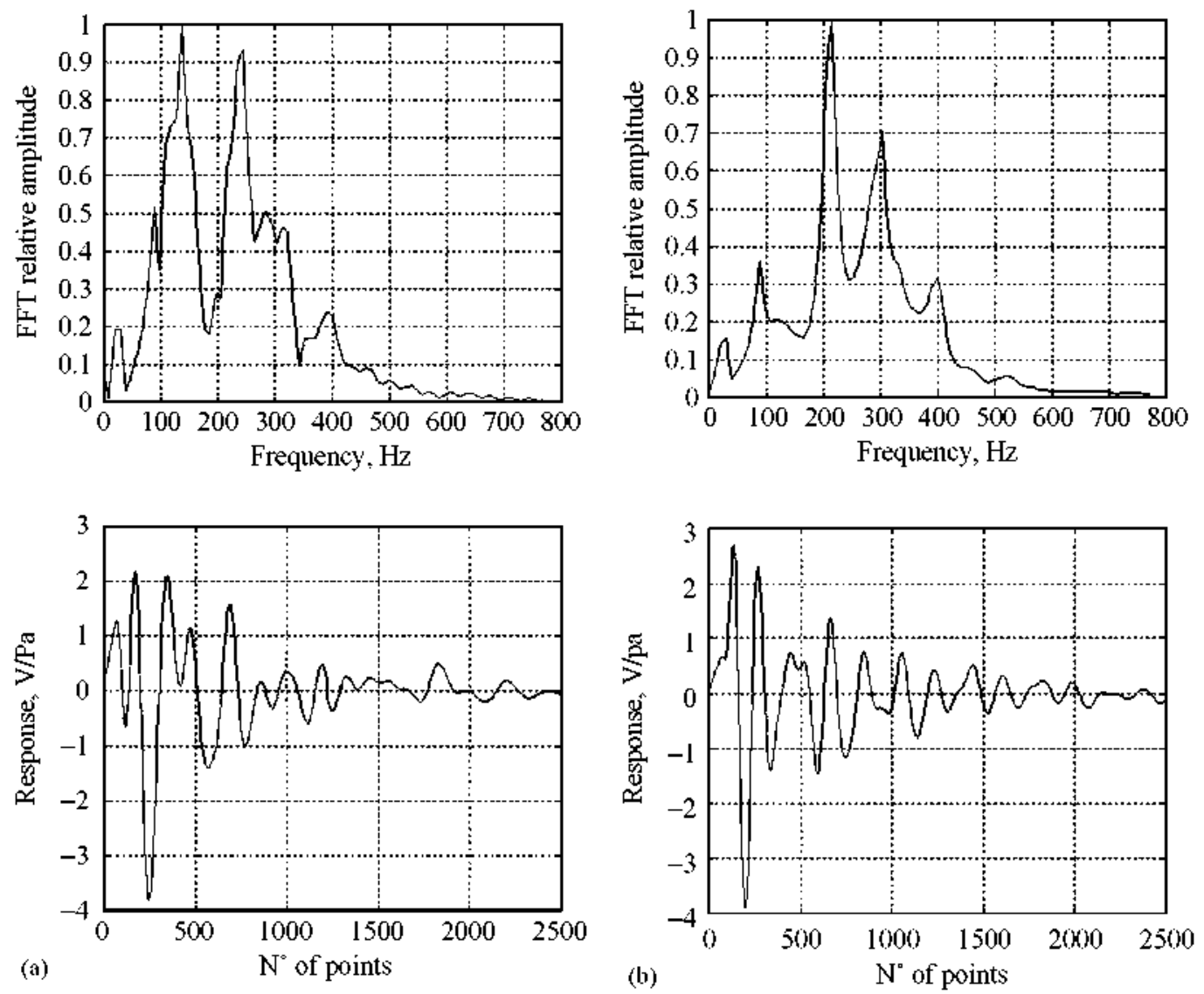

Fig. 2. The digitised acoustic impulse response, and the frequency spectra obtained by a fast Fourier transfom (FFT) for: (a) hollow watermelon; (b) good watermelon

the sum of the spectrum magnitudes between 0 and $500 \mathrm{~Hz}$ was defined first by Farabee (Farabee et al., 1991). In our research the $\mathbf{B M}_{n}$ were calculated by summing the normalised spectrum magnitude between two different frequencies. The bandwidths associated with the BM were based on the previous study by Farabee (Table 1).

The acoustic response of each watermelon was measured by hitting the fruit with an impactor and detecting the output sound by a microphone on the opposite side.

The impactor was made of a metal ball $(13 \mathrm{~g})$ fixed on a pendulum which was dropped onto the watermelon surface from a height of $120 \mathrm{~mm}$. A support block was formed by creating a shallow hollow on the upper side of a wooden block. A microphone, preamplifier and headphone were imbedded within the base of hollow and padding material inserted. The microphone was at a distance of $2-5 \mathrm{~mm}$ from the fruit and detected the impulse acoustic response (Fig. 3). The headphone insulated the microphone area while the padding material provided the necessary free supporting conditions.
Table 1

Frequency limits for BM analysis

\begin{tabular}{lc}
\hline BM identifier & Frequency limits, $\mathrm{Hz}$ \\
\hline $\mathrm{BM}_{1}$ & $85-160$ \\
$\mathrm{BM}_{2}$ & $40-90$ \\
$\mathrm{BM}_{3}$ & $60-110$ \\
$\mathrm{BM}_{4}$ & $70-120$ \\
$\mathrm{BM}_{5}$ & $80-130$ \\
$\mathrm{BM}_{6}$ & $100-180$ \\
$\mathrm{BM}_{7}$ & $120-200$ \\
\hline
\end{tabular}

Each watermelon was acoustically excited on two positions, both around the equator and rotated $90^{\circ}$. Nine measurements, divided in groups of three repetitions for the same location, together with three locations were performed at each position, therefore 18 measurements in each fruit. Positions identify the different areas of fruit where the impact is applied, locations indicate that the fruit is taken and relocated on the same position after some time, and repetitions refer to repeated data taken without changing or touching the fruit. 


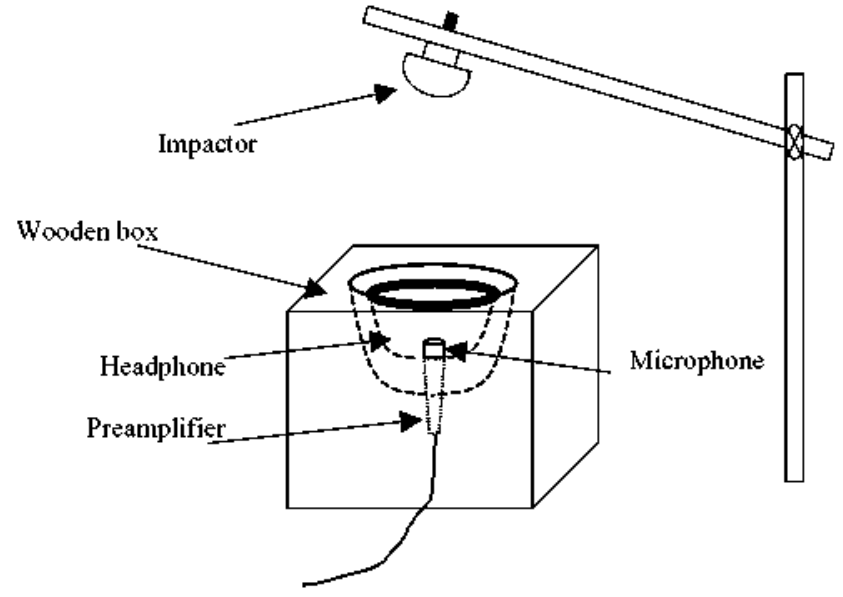

Fig. 3. Acoustic device: microphone position, impactor and structural elements

In addition to the acoustic tests, further nondestructive measurements were carried out: weight; characterisation of abnormal external shape; determination of maximum length of flower-stem axes and elastic parameters of intact fruit.

The elastic parameters were derived from compression of intact fruits with a semispherical indentor at the same place as previous acoustic measurements. The compression test was performed on a universal testing machine Texture Analyser XT2 (Stable Microsystems Inc.); a deformation of $2.5 \mathrm{~mm}$ was applied at $20 \mathrm{~mm} \mathrm{~min}^{-1}$. The maximum force in $\mathrm{N}$ required to attain such deformation was measured and the force/ deformation ratio in $\mathrm{N} / \mathrm{mm}$ calculated.

The density was gravimetrically measured by determining the mass and volume of the individual fruits by immersion in water, using Archimedes principle. Some differences were found between the density of hollow and good watermelons (Fig. 4). Owing to the size and shape of these fruits a large container is needed for the density measurements in laboratory; the volume determination was laborious and slow. For this reason, this procedure was rejected as a feasible solution for the industry.

Destructive tests were used to characterise the fruit flesh. The watermelons were cut in half lengthwise. Magness-Taylor penetration tests were carried out with the universal testing machine on the surface flesh of a half watermelon. The penetration tests were performed with an $8 \mathrm{~mm}$ diameter rod, a maximum penetration of $8 \mathrm{~mm}$ was applied at speed of $20 \mathrm{~mm} \mathrm{~min}^{-1}$. The maximum penetration force in $\mathrm{N}$ was registered. The positions of penetrations were fixed at a radial distance of $25 \mathrm{~mm}$ from the skin. Two equatorial diameters were measured. The soluble solids content was measured by a digital refractometer PR-101 ATAGO, on the same

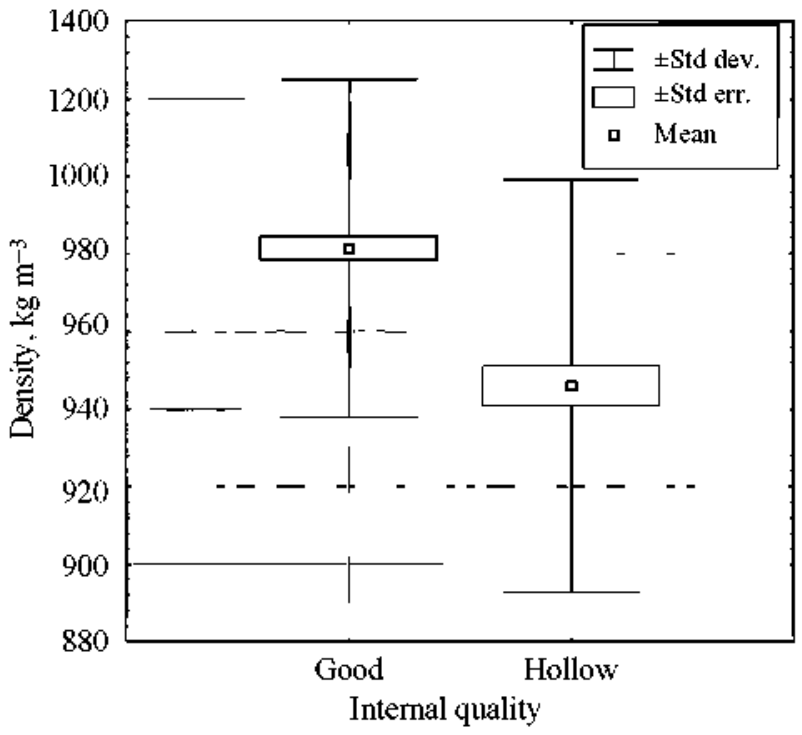

Fig. 4. Mean and standard deviation of density measured gravimetrically by determining mass and volume of fruit; volume is meastred by rotal immersion in water for yood and hollow watemelons, sample size $n=158$

position. The skin thickness was measured. Finally, a characterisation of internal creases or/and voids was carried out to determine: (1) size by the volume of the contained water; and (2) the position of the hollow with respect to the impact positions. Observations of ripeness, bruises and other internal disorders were also made.

\subsection{Materials}

Three experiments were designed: calibration, validation and overripeness study.

\subsubsection{Calibration experiment}

During a first testing season (2000), 158 seedless watermelons cv. 'Reina de Corazones' were studied in the laboratory. Watermelons were grown in commercial greenhouses as well as in open fields in Almería and Valencia, the main production areas in Spain. Several batches of fruits were harvested at commercial maturity and sent to the laboratory for the measurements during the production period (April-September). Human experts were used to classify the individual fruit in order to obtain balanced samples of good and hollow heart watermelons. However, some human classification errors and production characteristics (not hollow in some batches) resulted in the analysis of 105 good watermelons and 53 cracked watermelons. The hollow volume ranged from 5 to $1400 \mathrm{ml}$. 
All instrument measurements above explained were applied at this experiment.

\subsubsection{Validation experiment}

During the second season (2001) the acoustic device and the classification criteria were validated with 499 fruits. A total of 421 watermelons were measured in two packing houses: 238 fruits in Almeria, and 183 fruits in Valencia. Additionally, 78 watermelons were tested in the laboratory.

The measurements carried out were: acoustic parameters; mass; the two equatorial diameters which include the impact points (i.e. positions); soluble solids content by a refractometer PR-101 ATAGO; and skin thickness. Voids/creases and any other internal disorders were also characterised. The tests of mechanical impact response in the 238 watermelons measured in Almería was similar that defined for the test during the first seasons. However, this test was modified for the fruits tested in Valencia. Thus, five impact positions were fixed around of equator, each position was impacted three times (three repetitions) without changing locations.

\subsubsection{Overripeness experiment}

A test designed to study the effect of overripeness was performed with the acoustic device in the laboratory. The maturation of a sample of 20 watermelons was followed over a period of 3 weeks. The acoustic tests were repeated in the laboratory at days 7,14 and 21 of shelf-life period.

\subsection{Data analysis and metrology}

Statistica Correlation analysis and one-way analysis of variance (ANOVA) were the methods used to select the best predictor for the internal disorders. Classification criteria were formulated.

The ANOVA analysis was performed in order to split down the total variation into isolated sources, which could be associated to the different factors in the experimental design. Also the variability distributions of the hollow group compared to the variability distribution of the sound watermelons was studied. As the number of hollow watermelons analysed was lower than the number of sound watermelons, the initial sample size available for the analysis was limited. To obtain equilibrated groups, 62 watermelons were randomly selected from the initial 158: 31 fruits with cracks or hollows and 31 deemed to be good.

It was useful to identify the variability due to the impactor and the recording system of the acoustic signal, and the variability due to the slight differences when locating the fruit. Therefore, a metrological study of the parameters involved in the acoustic device was carried out focusing on repeatability and reproducibility of $\mathrm{BM}_{1}$, the acoustic parameter with the best behaviour for hollow detection in watermelons.

For addressing repeatability three repetitions of the excitation, in the same position are used, whereas for reproducibility measurements the watermelon is three times replaced on the acoustic support at the same position (i.e. locations).

If the location is noted as $j(j=1,2$ and 3$)$ and the repetition is denoted as $i$ ( $i=1,2$, and 3), using the notation used by Feinberg (1999), each repetition can be expressed as follows:

$$
X_{i j}=\mu+L_{i}+e_{i j}
$$

where: $X_{i j}$ is the measured value of the acoustic parameter for repetition $i$ and replacement or location $j ; \mu$ is the true value of the acoustic parameter for the considered position; $L_{j}$ is the bias due to the replacement $j ;$ and $e_{i j}$ is the random error of the measurement. Following the calculation of Feinberg, the inter-locations variance is denoted as $s_{L}^{2}$; it can be assumed that the variance of the repeatability is the intra-locations variance $s_{r}^{2}$. Assuming a normal distribution of the measurements, it can be proved that the variance of reproducibility, $s_{k}^{2}$ is equal to the sum of the interlocations and the repeatability variances:

$$
s_{R}^{2}=s_{L}^{2}+s_{r}^{2}
$$

Expanding each term:

$\sum_{i=1}^{r} \sum_{j=1}^{l}\left(x_{i j}-\overline{\bar{x}}\right)^{2}=\sum_{i=1}^{r}\left(\overline{x_{i}}-\overline{\bar{x}}\right)^{2}+\sum_{i=1}^{r} \sum_{i=1}^{l}\left(x_{i j}-\bar{x}_{i}\right)^{2}$

The variances $s_{I}^{2}$ and $s_{r}^{2}$ are obtained by dividing the two members of Eqn (3) by the corresponding degrees of freedom.

In this study, the repeatability and reproducibility calculation were computed for 20 different watermelons, using the data of each position in a watermelon as independent measurements: 10 hollow fruits and 10 good ones, selected randomly among one set of 122 . Each combination of watermelon and position is noted with the subscript $k$. The hollow watermelons used in the metrological study sample presented a hollow volume from 36 to $652 \mathrm{ml}$.

The mean of all the values for $s_{r}$ (number of samples $n=40$ ) was considered the repeatability error of the acoustic device, and the mean of all the values for $s_{R}$ was considered the reproducibility error of the measurement 
procedure:

$$
\begin{gathered}
\overline{s_{r}}=\frac{\sum_{k=1}^{n} s_{r k}}{n} \\
\overline{s_{R}}=\frac{\sum_{k=1}^{n} s_{R k}}{n}
\end{gathered}
$$

Identical calculations were carried out considering separately the two groups: hollow and good watermelons.

\section{Results and discussion}

\subsection{Calibration experiment}

A breakdown and one-way ANOVA analysis shows that the acoustic parameter $\mathrm{BM}_{1}$ is the best predictor for the internal hollows, according to its highest $F$-value $(F=133.51$; see Table 2$)$. The values for $\mathrm{BM}_{1}$ increase when internal voids appear in the watermelons. The representation of average and standard deviation for $\mathrm{BM}_{1}$ (Fig. 5) facilitated the formulation of a simple segregating criterion: watermelons with the average of the 18 values for $\mathrm{BM}_{1}$ larger than 25 are classified as hollow fruits. This criterion allowed $82.3 \%$ of the fruit to be correctly classified. Discriminant analysis on this data sets the optimal segregating threshold to $29 \cdot 6$, however classification is not improved.

A correlation analysis for hollow volume of all the acoustic parameters and the reference measurements is performed. The BM parameters provide the highest correlation values: from 0.5 to 0.7 . (Table 3 ).

To verify the ability of BM parameters to segregate watermelons with internal voids, a non-supervised classification was carried out. All averages of BM parameters were introduced in a cluster analysis, in our case a tree clustering. The purpose was to determine how the cases (or fruits) were organised according to its

Table 2

Analysis of variance (ANOVA) results (probability $P<0 \cdot 05$ ) for set calibration; factor is internal quality of watermelon (sound or hollow fruits); degree of freedom effect, 156

\begin{tabular}{lr}
\hline & F-value \\
\hline Resonant frequency & 3.97 \\
Maximum intensity & 17.29 \\
$\mathrm{BM}_{1}$ & 133.51 \\
$\mathrm{BM}_{2}$ & 60.71 \\
$\mathrm{BM}_{3}$ & 91.10 \\
$\mathrm{BM}_{4}$ & 108.58 \\
$\mathrm{BM}_{5}$ & 127.00 \\
$\mathrm{BM}_{6}$ & 115.80 \\
$\mathrm{BM}_{7}$ & 68.14 \\
\hline
\end{tabular}

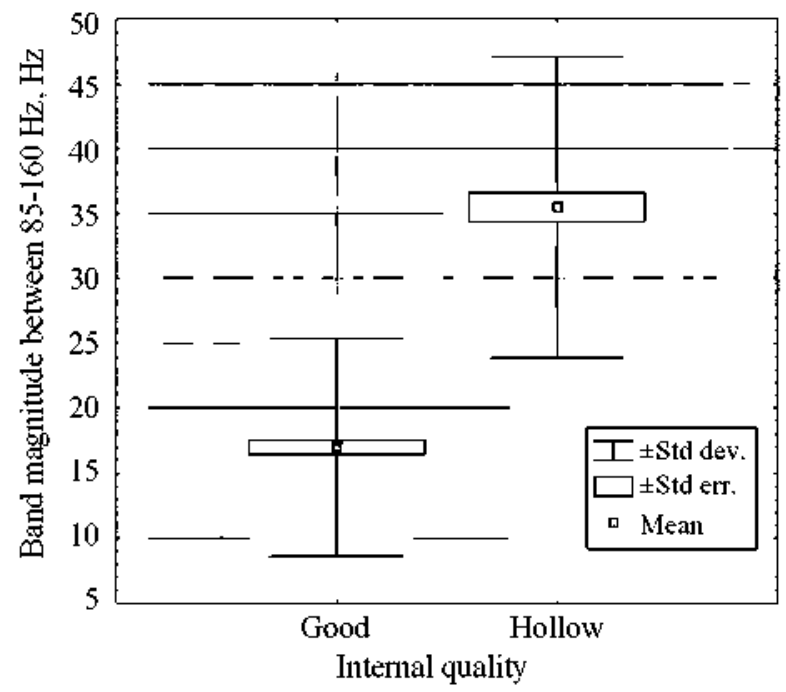

Fig. 5. Mean and standard deviation of the BM in the frequency ranges $85-160 \mathrm{~Hz}\left(\mathrm{BM}_{I}\right)$ for good and hollow waternelons, sample size $n=158$

Table 3

Correlation coefficients between acoustic and reference parameters and hollow volume

\begin{tabular}{lc}
\hline Parameters & Correlation coefficien \\
\hline Resonant frequency & -0.06 \\
Maxima intensity & -0.32 \\
Band magnitude & \\
$85-160 \mathrm{~Hz}\left(\mathrm{BM}_{1}\right)$ & 0.62 \\
$40-90 \mathrm{~Hz}\left(\mathrm{BM}_{2}\right)$ & 0.64 \\
$60-110 \mathrm{~Hz}\left(\mathrm{BM}_{3}\right)$ & 0.66 \\
$70-120 \mathrm{~Hz}\left(\mathrm{BM}_{4}\right)$ & 0.67 \\
$80-130 \mathrm{~Hz}\left(\mathrm{BM}_{5}\right)$ & 0.54 \\
$100-180 \mathrm{~Hz}\left(\mathrm{BM}_{6}\right)$ & 0.57 \\
$120-200 \mathrm{~Hz}\left(\mathrm{BM}_{7}\right)$ & 0.49 \\
Skin thickness & 0.18 \\
Soluble solids & 0.36 \\
Mass & 0.24 \\
Magness-Taylor force & -0.02 \\
Slope compression & -0.05 \\
Density & -0.28 \\
Cross-section & 0.2 \\
\hline
\end{tabular}

averages of the BMs. The tree clustering uses the Euclidean distance between cases to form the groups. The population (158 watermelons) was by this procedure divided into two main groups (Fig. 6). The averages of hollow volume were significantly different between both groups (1 and 2). Both groups each contained two sub-groups with a remarkable linkage distance among them. Averages of volume and weight for each group are shown in Table 4 . A trend is observed: the appearance of voids and creases occur more frequently for the largest watermelons (Table 5). 


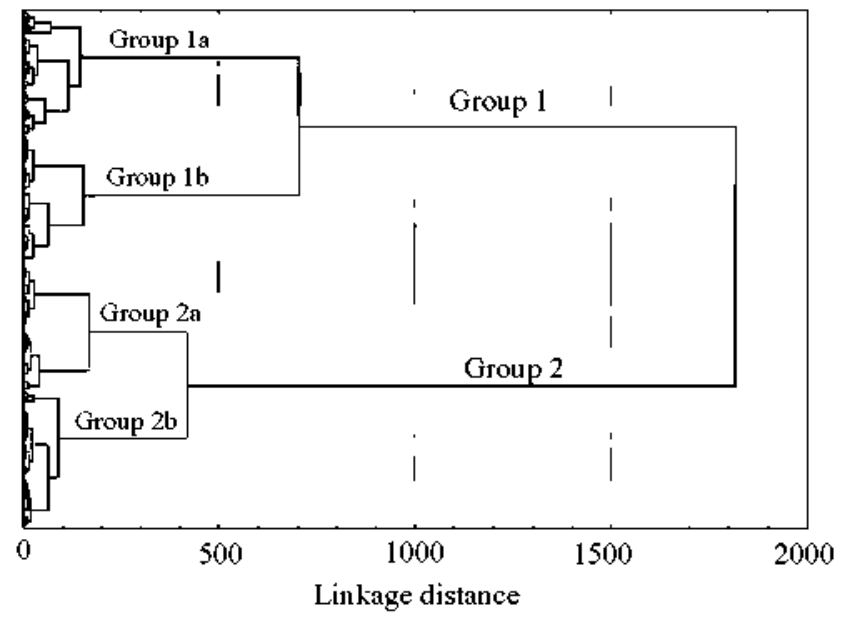

Fig 6. Tree diagram of cluster analvsis for 158 watermelons; variables in the analysis: $B M_{x}$ parameters, from $x=1-7$

Table 4

Averages in hollow volume and weight for each group obtained by unsupervised cluster analysis based on all BMs

\begin{tabular}{lcc}
\hline Cluster & Hollow tolume, $\mathrm{ml}$ & Mass, $\mathrm{kg}$ \\
\hline Subgroup 1a & 327.5 & 6.01 \\
Subgroup 1b & 45.5 & 5.7 \\
Group 1 & 184.57 & 5.85 \\
Subgroup 2a & 0 & 4.9 \\
Subgroup 2b & 23.7 & 5.05 \\
Group 2 & 13 & 4.98 \\
\hline
\end{tabular}

Table 5

Percentages of classification in the different Bayesian analyses. BM included in each analysis are marked

\begin{tabular}{lccc}
\hline & \multicolumn{3}{c}{ Classification, \% } \\
\cline { 2 - 4 } Sample & Wrong & Marginal & Correct \\
\hline Analysis 1: $\mathrm{BM}_{1}$ & 12 & 6.9 & $81 \cdot 1$ \\
Analysis 2: $\mathrm{BM}_{1}, \mathrm{BM}_{5}$ & $10 \cdot 7$ & 12 & 77.3 \\
Analysis 3: all BM & $10 \cdot 1$ & $10 \cdot 7$ & $79 \cdot 2$ \\
Analysis 4: $\mathrm{BM}_{2}, \mathrm{BM}_{3}$, & & & \\
$\mathrm{BM}_{4}, \mathrm{BM}_{5}, \mathrm{BM}_{5}, \mathrm{BM}_{7}$ & 8 & $15 \cdot 2$ & 76.8 \\
\hline
\end{tabular}

A Bayesian classifier was performed using the $\mathrm{BM}_{1}$ parameter. Each watermelon was characterised by the averages of the $18 \mathrm{BM}_{1}$ values produced in the impact positions. The probability $p_{1}$ and $p_{2}$ of any value, belonging to either of the two groups is calculated, on the basis of the normal distribution equations:

$$
p_{1}(x)=\frac{f_{1}(x)}{f_{1}(x)+f_{2}(x)} \quad \text { and } \quad p_{2}(x)=\frac{f_{2}(x)}{f_{1}(x)+f_{2}(x)}
$$

where $f_{1}(x)$ refers to the density probability function for class $i$ defined by means of its average and standard deviation.

Whenever the probability of a case to belong to group 1 (hollow units) is higher than $0 \cdot 6$, the watermelon is classified as a hollow. Conversely, the probability of a case to belong to group 2 (good watermelons) is higher to 0.6 , then the case is classified as a good watermelon. In any other case, the fruit is considered to be a marginal individual. Within this procedure, the proportion of correctly classified was $81.1 \%$, while the proportion of marginal cases was $6.9 \%$.

Further Bayesian classifiers were attempted using several BM parameters. For any of the Bayesian analysis frontiers cases increase whenever the number of BM parameters is increased, and so no better solution is achieved when increasing the number of variables in the analysis compared to the showed one.

Correlation coefficients between acoustic parameters and destructive ripeness indicators (soluble solids content and Magness-Taylor parameters), are very low. Nevertheless, watermelons showing extreme overripeness and/or bruised tissues at visual inspection also show high $\mathbf{B M}_{1}$ values. It may be concluded that these internal problems affect the acoustic parameters values in the same way as the presence of cracks or hollows.

\subsection{Validation experiment}

Tests are performed to validate results obtained during the first season. Concerning the classification criteria formulated earlier, two classes are considered: watermelons with hollow volume larger than $0 \mathrm{ml}$, and watermelons of hollow volume equal to $0 \mathrm{ml}$. The results of the validation test for the 183 fruits from Almeria showed an $81.5 \%$ of well-classified watermelons, according to the average $\mathrm{BM}_{1}$ values from the two impact points combined with the simple classification criterion. A $76.3 \%$ of well classified was obtained regarding the Bayesian criterion.

A comparison between the classification by a human expert (expert classification) and the acoustic device classification is carried out on 183 watermelons (out of the 499 tested), chosen from commercial batches regarding size variability (Table 6). When the simple classification criterion is used to segregate by means of the highest average $\mathrm{BM}_{1}$ value at any impact point (worst situation), a $78.14 \%$ of well-classified fruits is found (see Cell 1 of Table 6 ) by the acoustic device testing. If the average $\mathbf{B M}_{1}$ instead of the maximum is considered, the percentage of well-classified watermelons increases to $83.6 \%$, compared to $71.5 \%$ of human experts. This improvement in the classification is due to 
Table 6

Comparison of classification by a human expert versus the acoustic device in 183 watermelons tested in the second season; the simple classification criterion is used to segregate by means of the highest average $\mathrm{BM}_{1}$ value at any impact point; analysis for integritywatermelons divided into whole and hollow; analysis for quality-watermelons divided into sound internal quality and those with internal disorders, such as hollows, bruises or overripeness

\begin{tabular}{|c|c|c|c|c|c|c|}
\hline & \multicolumn{3}{|c|}{ Predicted integrity } & \multicolumn{3}{|c|}{ Predicted quality } \\
\hline & Whole fruit & Defective fruit & $\begin{array}{l}\text { Correctly clas- } \\
\text { sified, } \%\end{array}$ & Whole fruit & Defective fruit & $\begin{array}{l}\text { Correctly clas- } \\
\text { sified, } \%\end{array}$ \\
\hline $\begin{array}{l}\text { Monitored by human } \\
\text { expert }\end{array}$ & & & $71 \cdot 58$ & & & $82 \cdot 51$ \\
\hline Whole fruit & 78 & 41 & & 78 & 21 & \\
\hline Defective fruit & 11 & 53 & & 11 & 23 & \\
\hline $\begin{array}{l}\text { Monitored by acoustic } \\
\text { device }\end{array}$ & & & $78 \cdot 14$ & & & 89.07 \\
\hline Whole fruit & 87 & 32 & & 87 & 12 & \\
\hline Defective fruit & 8 & 56 & & 8 & 76 & \\
\hline
\end{tabular}

Table 7

Averages and standard deviation (SD) for $\mathrm{BM}_{1}$ in 20 watermelons stored during a 3 weeks period

\begin{tabular}{ccc}
\hline & \multicolumn{2}{c}{ Band magnitude } \\
\cline { 2 - 3 } Storage day no. & Average & Standard deviation \\
\hline 7 & 21.02 & 7.06 \\
14 & 23.35 & 8.73 \\
21 & 26.12 & 11.25 \\
\hline
\end{tabular}

the decrease of the good watermelons wrongly classified if the highest value for $\mathrm{BM}_{1}$ is considered.

An important feature is found when analysing the errors in both human experts and the instrumental device. The same 20 watermelons without hollows are wrongly classified by both methods. The internal quality of these 20 watermelons is studied. All the watermelons show bruises and/or overripeness. This fact confirms the hypothesis that the acoustic system is also able to detect these internal misquality factors. It was then decided not to consider these individuals as sound fruits. In such case the percentage of well-classified watermelons increases to $94.5 \%$ for the acoustic device testing and to $82.5 \%$ for the human expert.

\subsection{Overripeness study}

As a general feature, the average $\mathrm{BM}_{1}$ increases with shelf life (Table 7 and Fig. 7). However, the $\mathrm{BM}_{1}$ values for some watermelons were remained retained during storage. As a conclusion it is necessary to define an adequate reference tests and/or sensory descriptors in order to address the overripeness threshold for the acoustic system.

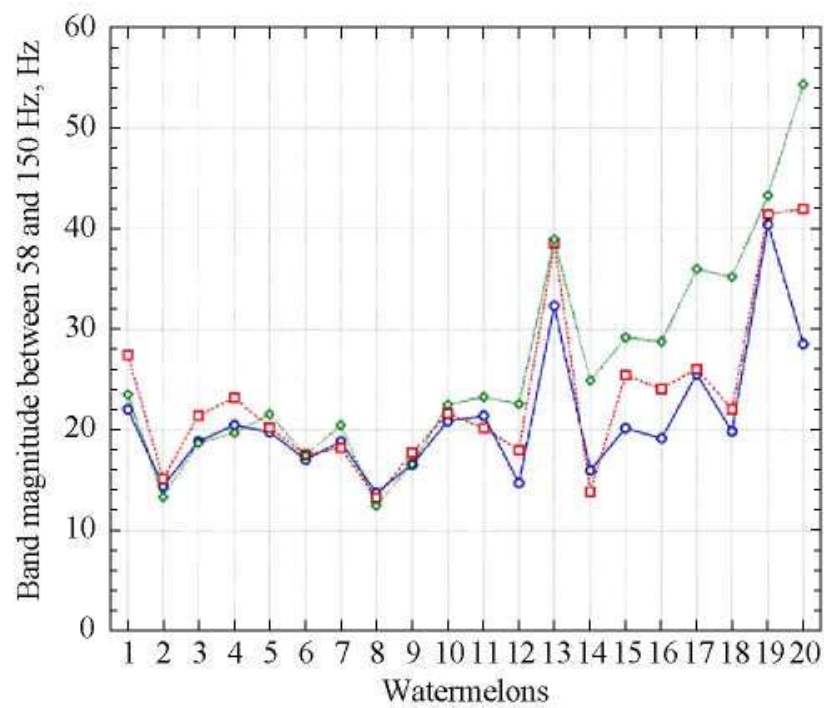

Fig. 7. Development of band magnitude $\left(B M_{1}\right)$ for watermelons stored for 3 weeks under shelf-life conditions; each number refers to one fruit; symbols indicate increase shelf-life period; only certain watermelons evolve under shelf-life conditions: $\bigcirc$, day 7;

$$
\square \text {, day } 14 ; \bullet \text {, day } 21
$$

\subsection{Metrological measurements}

The robustness of the application of the acoustic device to detect internal quality problems in the watermelon was studied through repeatability and reproducibility of the $\mathrm{BM}_{1}$ acoustic parameter. The values of repeatability error and reproducibility error are shown in Table 8 .

The instrumental error is assimilated as the repeatability error. Whatever the fruit and its internal quality state, the repetitions give close values: the standard deviation computed on three repetitions is no higher than 2.9 . The overall repeatability is $0.99,0.95$ in good 
watermelons and 1.03 in hollow ones, not significant differences were shown between the values of both groups in the ANOVA analysis.

The overall reproducibility computed as explained before was $2 \cdot 65$. The reproducibility error $\overline{s_{R}}$, shows significant differences between the good and the hollow groups of watermelon: 1.79 and 3.5 , respectively. The first value can be considered in accordance to the repeatability error obtained for good fruits, however, there is a large difference between the repeatability error and the reproducibility error for the hollow fruits. Therefore, small variations introduced at the locating of

Table 8

Values of the repeatability and reproducibility errors for the acoustic parameter $\mathrm{BM}_{1}$ considering separately good and hollow watermelons; 10 hollow and 10 sound watermelons were used, respectively

\begin{tabular}{lcc}
\hline Waternelon sample & $\begin{array}{c}\text { Repeatability } \\
\text { error, } \overline{s_{r}}\end{array}$ & $\begin{array}{c}\text { Reproducibility } \\
\text { error, } \overline{s_{R}}\end{array}$ \\
\hline $\begin{array}{l}\text { Hollow and good } \\
(n=20)\end{array}$ & 0.99 & 2.65 \\
Good $(n=10)$ & 0.95 & 1.79 \\
Hollow $(n=10)$ & 1.03 & 3.5 \\
\hline
\end{tabular}

$n$, sample number. the sample on the acoustic device produce higher measurement variability for hollow watermelons than for good watermelons. Still, the level of reproducibility is reasonable, $4.23 \%$ of the measured range of $\mathrm{BM}_{1}, 62$ units.

Metrological measurement related to reproducibility are consistent ANOVA analyses, as shown in Tables 9 and 10 , for good and for hollow watermelons, respectively. The mean square error is much higher in Table 10 (cracked watermelons). The highest $F$ value in both analyses corresponds to the watermelon effect $(586.88$ and 509.25). The effect position appeared as not significant for good watermelons, whereas in the hollow sample this effect is marked as significant. Also, the interaction effect between watermelon and position shows a $F$ value of 57.49 for hollow group, while remaining as 32.96 for watermelons without internal disorders. This fact points that the $\mathbf{B M}_{1}$ value is more affected by position for hollow than for sound watermelons.

This fact can be explained when considering the relative positions of the impact point and the internal crease. The $\mathrm{BM}_{1}$ values for the two measurement positions are in some cases very different for a single watermelon (Fig. 8). This situation occurs when the diameter defined by the impact point and the

Table 9

Analysis of variance (ANOVA) for $\mathrm{BM}_{1}$ for 31 watermelons without internal disorders; factors are watermelon, position (1,2) and replacement $(1,2,3)$; narked effects are significant with probability $P<0.05$ ( $\left.{ }^{*}\right)$ or with $P<0.01$ (**); degree of freedon of error, 372; mean square of error, 29.4

\begin{tabular}{|c|c|c|c|c|}
\hline Factor & Degree of freedom effect & Mean square effect & F value & Significance \\
\hline Watermelon & 30 & 1723.9 & 586.88 & *** \\
\hline Position & 1 & 2.7 & 0.92 & \\
\hline Replacement & 2 & 37.4 & 12.75 & $* *$ \\
\hline Watermelon position & 30 & 96.8 & 32.96 & $* *$ \\
\hline Watermelon replacement & 60 & $20 \cdot 5$ & 6.97 & $* *$ \\
\hline Position replacement & 2 & $22 \cdot 1$ & 7.53 & $* *$ \\
\hline All effects & 60 & 21.4 & $7 \cdot 30$ & $* *$ \\
\hline
\end{tabular}

Table 10

Analysis of variance (ANOVA) for $\mathrm{BM}_{1}$ for 31 hollow watermelons; factors are watermelon, position $(1,2)$ and replacenent $(1,2,3)$; marked effects are significant with probability $P<0.05(*)$ or with $P<0.01(* *)$; degree of freedom of error, 372; mean square of error, $5 \cdot 31$

\begin{tabular}{|c|c|c|c|c|}
\hline Facror & Degree of freedom effect & Mean square effect & F value & Significance \\
\hline Watermelon & 30 & $2704 \cdot 2$ & 509.25 & $* *$ \\
\hline Position & 1 & 33.6 & $6 \cdot 32$ & $*$ \\
\hline Replacement & 2 & 37.2 & 7.00 & $* *$ \\
\hline Watermelon position & 30 & $305 \cdot 3$ & 57.49 & $* *$ \\
\hline Watermelon replacement & 60 & 55.6 & $10 \cdot 47$ & $* *$ \\
\hline Position replacement & 2 & $11 \cdot 3$ & $2 \cdot 14$ & \\
\hline All effects & 60 & 46.8 & 8.82 & $* *$ \\
\hline
\end{tabular}




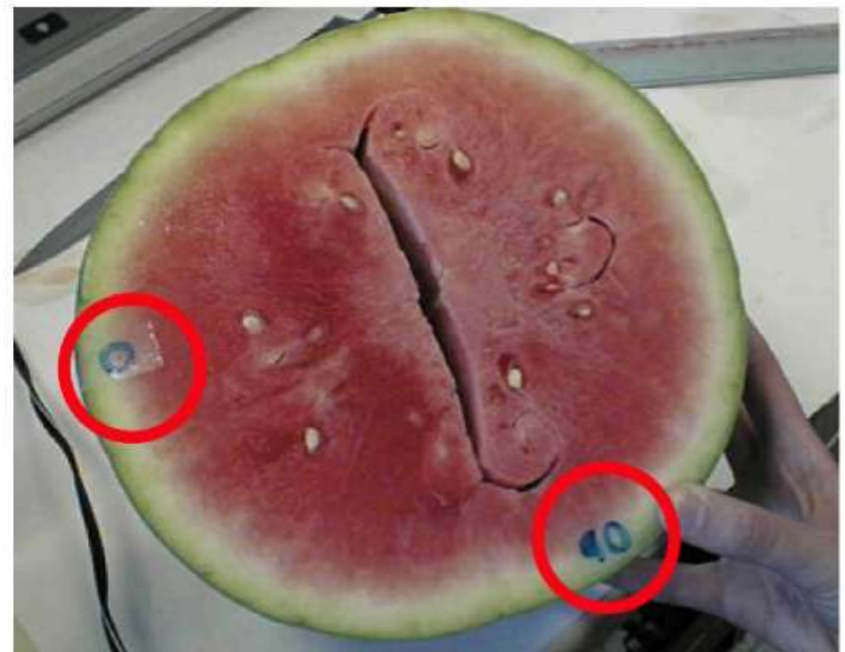

Fig. 8. Hollow watermelon (hollow volume $=100 \mathrm{ml}$ ) with very different $B M_{1}$ values in the two impact position; the mean value of the $B M_{1}$ was 38.9 when the fruit was excited in the impact point called ' $O$ ' and 16.8 when it was excited in ' 90 '

microphone crossed the void area and excited it, the BM value increased. Therefore, when applying the acoustic measurement in two or more different positions around the equator of each watermelon a decrease in the percentage of 'false negatives', is obtained; false negative stands for hollow watermelons classified as good ones.

\section{Conclusions}

The results show that the non-destructive acoustic response device can be used to classify watermelons into quality classes according to their internal state regarding the presence of creases and hollows in the flesh. Further evidence was obtained for the detection of bruises and overripeness as well. The BM parameters, obtained by summing the normalised spectrum magnitude between two determined frequencies, were the best indicators of internal quality, especially the BM encompassed between 85 and $160 \mathrm{~Hz}\left(\mathrm{BM}_{1}\right)$, which showed the minimum overlap between good and hollow watermelons. A simple classification criterion based on the setting of a $\mathrm{BM}_{1}$ threshold value, showed the best percentages of success. The repeatability error of the acoustic measurements presents acceptable levels. The reproducibility error includes a variability due to the internal state of the watermelon. Thus higher variability is observed in the hollow sample because of the flesh heterogeneity.

In order to detect the internal crack in watermelons at least two impact points around equator are recom- mended. Due to the lack of homogeneity in the distribution of disorders inside the fruits, a different frequency response is expected when exciting at different places on the object. The best results are been obtained when five places on the surface of the fruits are excited and the averages values for $\mathrm{BM}_{1}$ per watermelons are computed.

\section{Acknowledgements}

We thank the Spanish Ministry of Science and Technology for financing the national project (PETRI 95-0376-OP), and the Community of Madrid for funding.

\section{References}

Armstrong P R; Zapp H R; Brown G K (1990). Impulsive excitation of acoustic vibrations in apples for firmness determination. Transactions of the ASAE, 33, 1353-1359

Armstrong P R; Stone M L; Brusewitz G H (1997). Peach firmness determination using two different nondestructive vibrational sensing instruments. Transactions of the ASAE, 40, 699-703

De Baerdemaeker J; Lemaitre L; Meire R (1982). Quality detection by frequency spectrum analysis of the fruit impact force. Transactions of the ASAE, 25, 175-78

De Belie N; Schotte S; Lammertyn J; Nicolai $B$; De Baerdemaeker J (2000). Firmness changes of pear fruit before and after harvest with the acoustic impulse response technique. Journal Agricultural Engineering Research, 77, 183-191

Farabee M; Stone M L (1991). Determination of watermelon maturity with sonic impulse testing. ASAE Paper No. 913013

Feinberg M (1999). L'assurance Qualité dans les Laboratoires Agroalimentoires et Pharmaceutiques. Quality Assurance in Food and Pharmaceutical Laboratories. Editions TEC \& DOC, Paris

Kim J T; Stubbs N (2003). Crack detection in beam-type structures using frequency data. Journal of Sound and Vibration, 259(1), 145-160

Maynard D N; Hopkins D L (1999). Watermelon fruit disorders. HortTechnology, 9(2), 155-161

Silva J M; Gomes J L (1994). Crack identification of simple structural elements through the use of natural frequency variations: the inverse problem. 12th International Modal Analysis Conference, Honolulu, pp. 1728-1735

Stone M L; Armstrong P R, Zhang X; Brusewitz G H; Chen D D (1996). Watermelon maturity determination in the field using acoustic impulse impedance techniques. Transactions of the ASAE, 39, 2325-2330

Stubbs N; Osegueda R (1990). Global nondestructive damage evaluation in solids. International Journal of Analytical and Experimental Modal Analysis, 5, 67-69 